\title{
Laser doppler spectrum decomposition applied in diagnostics of microcirculatory disturbances
}

Igor O. Kozlov, Evgeny A. Zherebtsov, Victor V. Dremin, Angelina I. Zherebtsova, Elena V. Zharkikh, et al.

Igor O. Kozlov, Evgeny A. Zherebtsov, Victor V. Dremin, Angelina I. Zherebtsova, Elena V. Zharkikh, Andrey V. Dunaev, Edik U. Rafailov, "Laser doppler spectrum decomposition applied in diagnostics of microcirculatory disturbances," Proc. SPIE 10685, Biophotonics: Photonic Solutions for Better Health Care VI, 1068540 (17 May 2018); doi: 10.1117/12.2289551

SPIE. Event: SPIE Photonics Europe, 2018, Strasbourg, France 


\title{
Laser Doppler spectrum decomposition applied in diagnostics of microcirculatory disturbances
}

\author{
Igor O. Kozlova ${ }^{\mathrm{a}}$, Evgeny A. Zherebtsov ${ }^{\mathrm{b}}$, Viktor V. Dremin ${ }^{\mathrm{a}}$, Angelina I. Zherebtsova ${ }^{\mathrm{a}}$, \\ Elena V. Zharkikh ${ }^{\mathrm{a}}$, Andrey V. Dunaev ${ }^{\mathrm{a}}$, and Edik U. Rafailov ${ }^{\mathrm{b}}$ \\ ${ }^{a}$ Orel State University named after I.S. Turgenev, Komsomolskaya St. 95, Orel, Russia \\ ${ }^{\mathrm{b}}$ Aston Institute of Photonic Technologies, Aston University, Aston Triangle, Birmingham, UK
}

\begin{abstract}
Laser Doppler flowmetry (LDF) is widely used to study blood microcirculation in the skin. However, during tradition signal processing based on the integral estimations of the power spectrum of detector photocurrent, the significant part of the information about the skin blood flow is lost. In this study, we propose to analyse the distribution of the blood perfusion over the Doppler shift frequencies, which correlate with the RBC velocity. This approach provides localisation of the blood flow oscillations in different subranges of the Doppler shift. The method applied together with the wavelet analysis has been tested in healthy volunteers and patients with psoriasis on the unaffected surface of the skin. It was revealed, that the significant difference in the amplitude of myogenic oscillations is allocated in the region of the low frequency Doppler shift (1-200 Hz). This frequency region can be associated with the signal from slow components of the skin microcirculation, that can point out on a different state of the lymphatic system of the skin in psoriasis.
\end{abstract}

Keywords: Laser Doppler flowmetry, Doppler spectrum decomposition, blood microcirculation, wavelet analysis, psoriasis

\section{INTRODUCTION}

The microcirculation system is an important transport system of the body, which delivers nutrients and take part in gas exchange. The state of this system directly affects the metabolism of the tissues and their functionality. At present, several methods of non-invasive diagnosis of the functional state of microcirculation have been developed: photoplethysmography, speckle-contrast imaging, optical coherence tomography, etc. In general case, these methods have different fields as well as aspects of the application. Laser Doppler flowmetry has relatively simple instrument implementation. One is the only method which can be implemented in compact wearable form factor and is sensitive to micro blood flow. Diagnostics significance of the method is in a wide range of diseases associated with microvascular disturbances (complications in diabetes mellitus, Raynaud's and other rheumatological diseases etc. $)^{1}$ Laser Doppler flowmetry is based on single mode infrared laser emission into the tissue with photodetection of back-scattered and Doppler-shifted on moving red blood cells radiation. ${ }^{2}$ After that, the power spectrum of received photocurrent is processed and analysed. The measuring parameter in this method is the perfusion unit $(P U)$, which is proportional to the concentration and velocity of RBC in diagnostic volume: ${ }^{3}$

$$
P U=\int_{f_{1}}^{f_{2}} f \cdot S(f) d f,
$$

where $S(f)$ - Doppler shift amplitude of power spectrum on frequency $f ; f_{1}, f_{2}$ - limits of the integration over frequencies of the registered spectrum. ${ }^{4}$ At a first approximation, the amplitude of power spectrum and frequency of Doppler shift are proportional to RBC concentration and velocity in diagnostic volume, consequently. ${ }^{5}$

Further author information: (Send correspondence to Kozlov Igor)

E-mail: igor57_orel@mail.ru Telephone: +7 9208140458

Biophotonics: Photonic Solutions for Better Health Care VI, edited by Jürgen Popp,

Valery V. Tuchin, Francesco Saverio Pavone, Proc. of SPIE Vol. 10685, 1068540

(C) 2018 SPIE - CCC code: 0277-786X/18/\$18 - doi: 10.1117/12.2289551

Proc. of SPIE Vol. 10685 106854O-1 
The existing instrumental implementations of the technique (Perimed Instruments, Moor Instruments, LAZMA) use signal processing algorithm with integration over a wide frequency range of Doppler shift, which does not allow for obtaining information about details of the perfusion distribution over the Doppler shift frequencies. In the study we propose a modification of the signal processing algorithm with the evaluation of the perfusion over the subranges (with step 100-200 Hz) of the Doppler shift. It was shown ${ }^{6,7}$ that functional tests (occlusion and local pressure) lead to redistribution of the blood flow to various frequency ranges of Doppler shift that can be interpreted as an alteration of $\mathrm{RBC}$ fraction with a certain velocity.

\section{MATERIAL AND METHODS}

For blood perfusion distribution to be registered we've developed LDF device with the possibility to register and save power spectra of the signal. ${ }^{8}$ Single-mode laser with a wavelength of $1064 \mathrm{~nm}$ was selected as a source of radiation for registration of blood perfusion. Fibre optical probe was used to deliver radiation to the skin and to collect backscattering light. Silicon photodetectors converted detected radiation into photocurrent. Signal processing unit consisted of the electronic board with the AC and DC amplifiers, analogue-to-digital conversion implemented using data acquisition board NI USB 6211. Finally, NI LabVIEW environment installed on PC was used for signal processing. An example of registered perfusion distribution during 10 minute blood perfusion measurements is shown in Figure 1a.

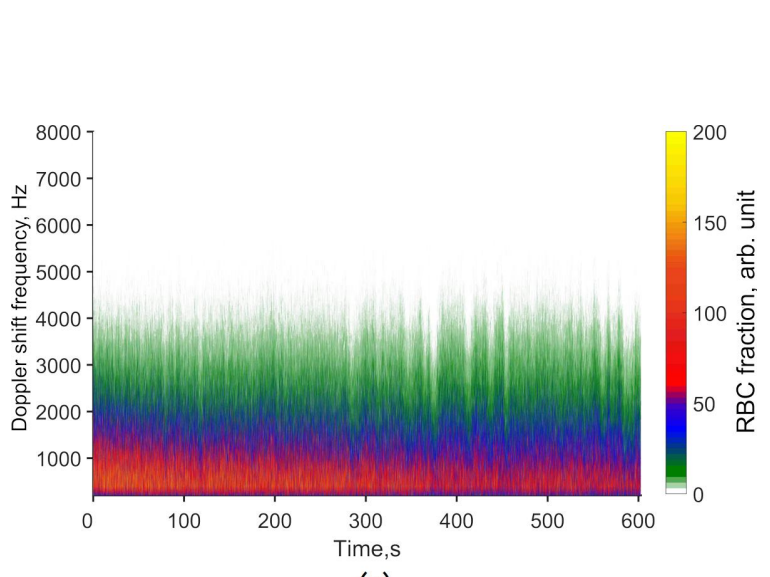

(a)

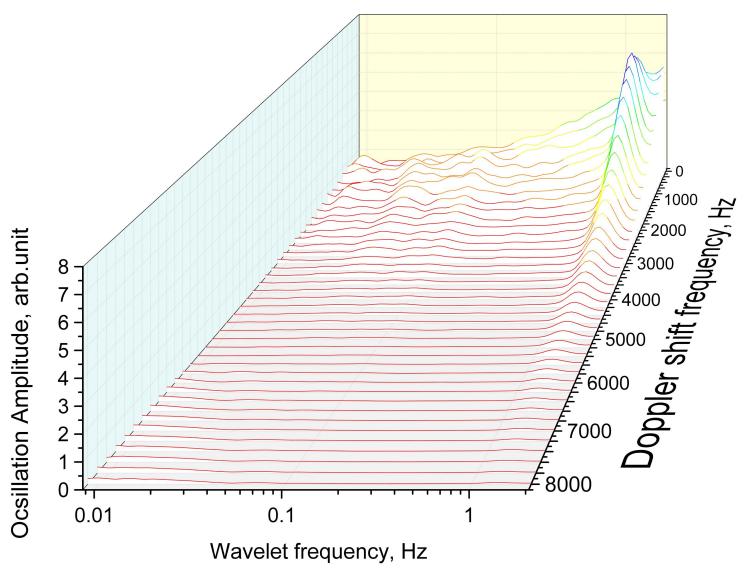

(b)

Figure 1. Example of perfusion distribution respectively to Doppler shift (a) and wavelet analysis blood perfusion subranges (b)

This approach is sensitive to the redistribution and changes in RBC velocity ${ }^{9}$ and concentration. ${ }^{10}$ This method provides the possibility to estimate the contribution of every subrange in general signal (Fig. 1b). The next stage of signal processing is wavelet analysis of the perfusion signal evaluated in Doppler shift frequency subranges. The wavelet analysis with Morlet core function is widely used in estimating of blood flow oscillations: ${ }^{11,12}$

$$
\psi(t)=e^{2 \pi i} \cdot e^{-t^{2} / 2 a^{2}}
$$

where $a=1$. Evaluated by the wavelength analysis localization of oscillations in subranges of Doppler shift has potential to be useful in diagnostics and physiological studies. In work we involved 5 patients with psoriasis $(2$ male, 3 female) and 5 healthy volunteers ( 3 male, 2 female) of the same age ( $23 \pm 2$ year-olds). The dorsal surface of the right forearm with non-lesion skin was chosen as an area of interest. The blood perfusion recordings were conducted with 10 minutes duration. At the next step of signal processing, registered perfusion distribution was divided into subranges (with the step of $100 \mathrm{~Hz}$ ) of Doppler shift frequency and integrated as frequency-resolved blood perfusion. The set of received time series underwent the wavelet analysis for localisation of the amplitude oscillations over the frequency of Doppler shift. Such representation (fig. 2) can help to reveal the most valuable subranges of Doppler shift frequencies with highest amplitudes of oscillations or with better sensitivity to a particular morbid condition. 


\section{EXPERIMENTAL RESULTS AND DISCUSSIONS}

As it represented on a Figure 1a, the main part of perfusion signal is localised below $2000 \mathrm{~Hz}$ of Doppler shift frequency. The conducted wavelet analysis of the blood perfusion records over the formed frequency subranges has revealed that the signal is significantly modulated in the relatively narrow bandwidth of frequency (Fig. 1b). Thus, for a specific applications sampling rate of the photocurrent digitising can be reduced. More narrow

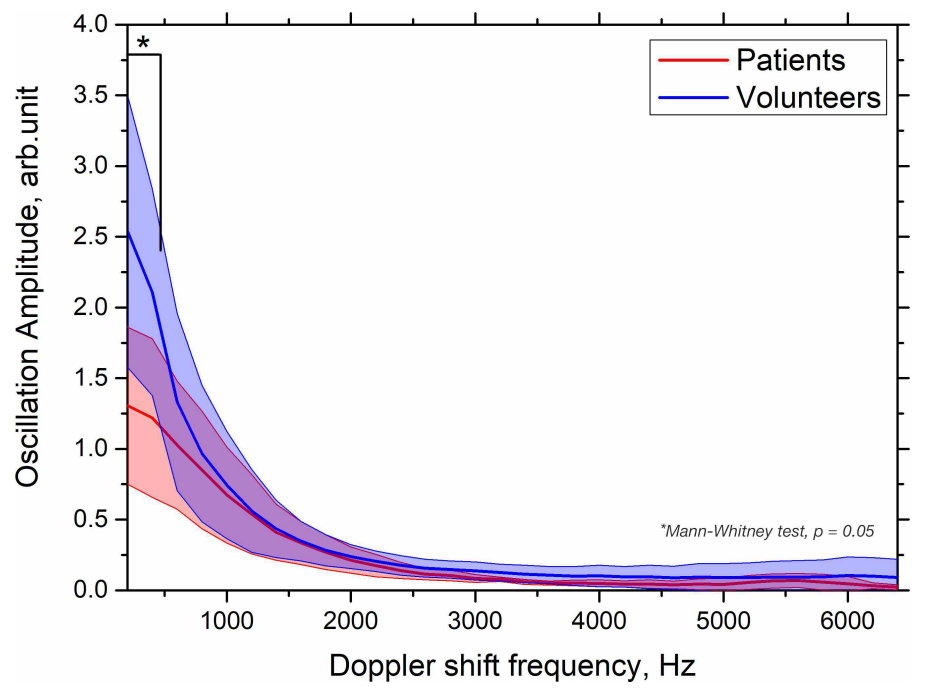

Figure 2. Amplitude distribution of myogenic oscillation of $0.1 \mathrm{~Hz}$ over the frequency of Doppler shift in patients and volunteers

spectral range of the Doppler shift registration can help to prolongate the work of wearable LDF-devices with less energy-consuming signal processing. The experimental results of the study in patients with psoriasis and volunteers demonstrated that amplitude distribution of myogenic oscillations are significantly varied over the Doppler shift frequency. According to Mann-Whitney test $(\mathrm{p}=0.05)$ the two first Doppler shift subranges of integration (0-100 and 100-200 Hz) has the statistically significant difference in this parameter whereas other subranges have been overlapped. This frequency region can be associated with the signal from slow components of the skin microcirculation. This result can point out on a different state of the lymphatic system of the skin in psoriasis even in the areas without visible lesions.

\section{CONCLUSIONS}

In this study, we examined the advantages of the analysis of the blood perfusion distribution over the Doppler shift frequencies. This approach provides localisation of the blood flow oscillations in different subranges of the Doppler shift. The method applied together with the wavelet analysis has been tested in healthy volunteers and in patients with psoriasis on the unaffected surface of the skin. It was revealed, that the significant difference in the amplitude of myogenic oscillations is allocated in the region of the low-frequency Doppler shift (1-200 Hz). By the example, it has been demonstrated that the use of flexible ranges of integration can significantly increase the sensitivity of the diagnostic parameters derived from the perfusion signal.

\section{ACKNOWLEDGMENTS}

Evgeny Zherebtsov kindly acknowledges funding from the European Union's Horizon 2020 research and innovation program under the Marie Sklodowska-Curie grant agreement No. 703145. The work has been funded by the grant of the President of the Russian Federation for state support of young Russian scientists MK-3400.2018.8, and by RFBR according to the research project 18-02-00669. 


\section{REFERENCES}

[1] Zherebtsov, E., Zherebtsova, A., Doronin, A., Dunaev, A., Podmasteryev, K., Bykov, A., and Meglinski, I., "Combined use of laser Doppler flowmetry and skin thermometry for functional diagnostics of intradermal finger vessels," Journal of Biomedical Optics 22(4) (2017).

[2] Nilsson, G. E., "Signal processor for laser doppler tissue flowmeters," Medical and Biological Engineering and Computing 22, 343-348 (Jul 1984).

[3] Dunaev, A. V., Zherebtsov, E. A., Rogatkin, D. A., Stewart, N. A., Sokolovski, S. G., and Rafailov, E. U., "Novel measure for the calibration of laser Doppler flowmetry devices," 8936, 89360D-89360D-7 (2014).

[4] Bonner, R. and Nossal, R., "Model for laser Doppler measurements of blood flow in tissue," Applied Optics 20(12), 2097-2107 (1981).

[5] Fredriksson, I., Larsson, M., and Strömberg, T., "Absolute flow velocity components in laser Doppler flowmetry," 6094, 60940A-60940A-12 (2006).

[6] Kozlov, I. O., Zherebtsov, E. A., Zherebtsova, A. I., Dremin, V. V., and Dunaev, A. V., "Investigation of Doppler spectra of laser radiation scattered inside hand skin during occlusion test," in [Journal of Physics: Conference Series], 929(1) (2017).

[7] Zherebtsov, E., Kandurova, K., Seryogina, E., Kozlov, I., Dremin, V., Zherebtsova, A., Dunaev, A., and Meglinski, I., "The influence of local pressure on evaluation parameters of skin blood perfusion and fluorescence," in [Progress in Biomedical Optics and Imaging - Proceedings of SPIE], 10336 (2017).

[8] Dremin, V. V., Zherebtsov, E. A., Makovik, I. N., Kozlov, I. O., Sidorov, V. V., Krupatkin, A. I., Dunaev, A. V., Rafailov, I. E., Litvinova, K. S., Sokolovski, S. G., and Rafailov, E. U., "Laser Doppler flowmetry in blood and lymph monitoring, technical aspects and analysis," 10063, 1006303-1006308 (2017).

[9] Volkov, M. V., Kostrova, D. A., Margaryants, N. B., Gurov, I. P., Erofeev, N. P., Dremin, V. V., Zharkikh, E. V., Zherebtsov, E. A., Kozlov, I. O., and Dunaev, A. V., "Evaluation of blood microcirculation parameters by combined use of laser Doppler flowmetry and videocapillaroscopy methods," 10336, 1033606-1033607 (2017).

[10] Wojtkiewicz, S., Wojcik-Sosnowska, E., Jasik, M., Maniewski, R., Karnafel, W., and Liebert, A., "Assessment of speed distribution of red blood cells in the microvascular network in healthy volunteers and type 1 diabetes using laser Doppler spectra decomposition," Physiological Measurement 35(2), 283 (2014).

[11] Stefanovska, A., Bracic, M., and Kvernmo, H. D., "Wavelet analysis of oscillations in the peripheral blood circulation measured by laser doppler technique," IEEE Transactions on Biomedical Engineering 46, 12301239 (Oct 1999).

[12] Makovik, I. N., Dunaev, A. V., Dremin, V. V., Krupatkin, A. I., Sidorov, V. V., Khakhicheva, L. S., Muradyan, V. F., Pilipenko, O. V., Rafailov, I. E., and Litvinova, K. S., "Detection of angiospastic disorders in the microcirculatory bed using laser diagnostics technologies," Journal of Innovative Optical Health Sciences 11(01), 1750016 (2018). 